\title{
Fully automatic scheme for measuring liver volume in 3D MR images
}

\author{
Trong-Ngoc Le ${ }^{\mathrm{a}, \mathrm{b}}$, Pham The Bao ${ }^{\mathrm{b}}$ and Hieu Trung Huynh, ${ }^{\mathrm{a},}$ \\ ${ }^{a}$ Faculty of Information Technology, Industrial University of Ho Chi Minh City, Viet Nam \\ ${ }^{\mathrm{b}}$ Faculty of Information Technology, University of Science, Ho Chi Minh City, Viet Nam
}

\begin{abstract}
In this paper, a fully automatic scheme for measuring liver volume in 3D MR images was developed. The proposed MRI liver volumetry scheme consisted of four main stages. First, the preprocessing stage was applied to T1weighted MR images of the liver in the portal-venous phase to reduce noise. The histogram of the 3D image was determined, and the second-to-last peak of the histogram was calculated using a neural network. Thresholds, which are determined based upon the second-to-last peak, were used to generate a thresholding image. This thresholding image was refined using a gradient magnitude image. The morphological and connected component operations were applied to the refined image to generate the rough shape of the liver. A 3D geodesic-active-contour segmentation algorithm refined the rough shape in order to more precisely determine the liver boundaries. The liver volumes determined by the proposed automatic volumetry were compared to those manually traced by radiologists; these manual volumes were used as a "gold standard." The two volumetric methods reached an excellent agreement. The Dice overlap coefficient and the average accuracy were $91.0 \pm 2.8 \%$ and $99.0 \pm 0.4 \%$, respectively. The mean processing time for the proposed automatic scheme was $1.02 \pm 0.08 \mathrm{~min}$ (CPU: Intel, core $\mathrm{i} 7,2.8 \mathrm{GHz})$, whereas that of the manual volumetry was $24.3 \pm 3.7 \mathrm{~min}(\mathrm{p}<0.001)$.
\end{abstract}

Keywords: Liver volumetry, MR volumetry, resection, transplantation, magnetic resonance imaging

\section{Introduction}

Over the past decades the field of transplantation has made great strides, leading to huge increases in survival rates. One assessment that is critical for a successful transplantation procedure is the estimation of the total and segmental liver volume; this calculation is indispensable because the liver size is one of the major predictors for the safe outcome of both the donor and recipient. Therefore, accurate and noninvasive liver volumetry is important in planning a liver transplantation [1-4]. Although the manual tracing of a liver boundary on each image can give accurate results, it has its limitations and is both subjective and time-consuming. It also has relatively large intra- and interobserver variations. Therefore, to address these issues, a number of schemes for computerized liver segmentation have been developed, and it has become an important research topic investigated by research groups worldwide.

To date, a number of researchers have developed schemes for computerized liver segmentation on CT images including feature analysis [5], image-processing techniques [6-8], region-growing [9-11],

\footnotetext{
* Address for correspondence: Hieu Trung Huynh, Faculty of Information Technology, Industrial University of Ho Chi Minh City, Viet Nam. Tel.: 84-94 268 2608; Fax: 84-838946268; E-mail: hthieu@ieee.org.
} 
shape constrained segmentation [12-15], rule-based segmentation [16], graph-cut [17], and level-set segmentation [18-19]. Compared to CT-based schemes, there have been fewer studies conducted on computerized liver segmentation for MR images despite there not being a risk of ionizing radiation. This discrepancy is likely because MR liver volumetry is believed to be more difficult and to have more variations than CT volumetry. The research that has been conducted in this field includes that of Karlo, et al. [20], whose work compared the CT- and MRI-based volumetry of resected liver specimens with intraoperative volume and weight measurements to calculate conversion factors. Furthermore, Farraher, et al. [21] developed a semi-automated dual-space clustering segmentation method. Their method required manually drawing a small region-of-interest (ROI) on the liver; then, it iteratively evaluated temporal liver segmentations with the repeated adjustment of parameters to obtain the final liver segmentation result. The performance of their method was evaluated on eighteen normal and nine abnormal cases. Ruskó, et al. [22] proposed a scheme to represent the liver based on a partitioned probabilistic model. In this method, the liver was partitioned into multiple regions, and different intensity statistical models were applied to these regions. This particular scheme's performance was evaluated on six abnormal and two normal cases. Gloger, et al. [23] developed a three-step segmentation method based on a region-growing approach, probability maps, and linear discriminant analysis. Their method was evaluated with twenty normal cases and ten fatty cases. In a previous study, the authors proposed a computerized scheme using the fast marching algorithm, and a geodesic active contour model. Its performance was evaluated on twenty-three cases [24].

The aforementioned studies showed significant promise; however, improving liver volumetry is still a priority. In this study, a fully automatic liver segmentation scheme in 3D MR images based on the thresholding method and geodesic active contour segmentation was developed. The performance of the proposed scheme was evaluated on ten cases, and a comparison between the proposed automatic volumetry and the gold-standard manual volumetry was performed.

\section{The fully automatic scheme for liver segmentation}

The proposed scheme for liver volumetry on a 3D MR image is shown in Figure 1. In order to reduce noise, an anisotropic diffusion filter is used [25]. This filter follows a differential equation called a modified curvature diffusion Equation [26]:

$$
\frac{\partial I}{\partial t}=|\nabla I| \nabla \cdot c(|\nabla I|) \frac{\nabla I}{|\nabla I|},
$$

where $\nabla$ is the gradient operator, and is the diffusion coefficient controlling the sensitivity of the edge contrast ( $K$ is a user-specified conductance parameter that was set to 2.0 in this scheme). This algorithm reduces the noise in the image while simultaneously preserving the major liver structures, such as major vessels and liver boundaries.

A gradient magnitude filter is applied to the reduced-noise images to enhance the boundaries and create an edge image. The scale of enhancing edges is controlled by a standard deviation of a Gaussian filter applied to the reduced image. The magnitude of the image gradient at each voxel is calculated by the following equation: 


$$
I_{M}=\sqrt{\left(\frac{\partial I_{G}}{\partial x}\right)^{2}+\left(\frac{\partial I_{G}}{\partial y}\right)^{2}+\left(\frac{\partial I_{G}}{\partial z}\right)^{2}}
$$

where $I_{G}$ is the Gaussian filtered image.

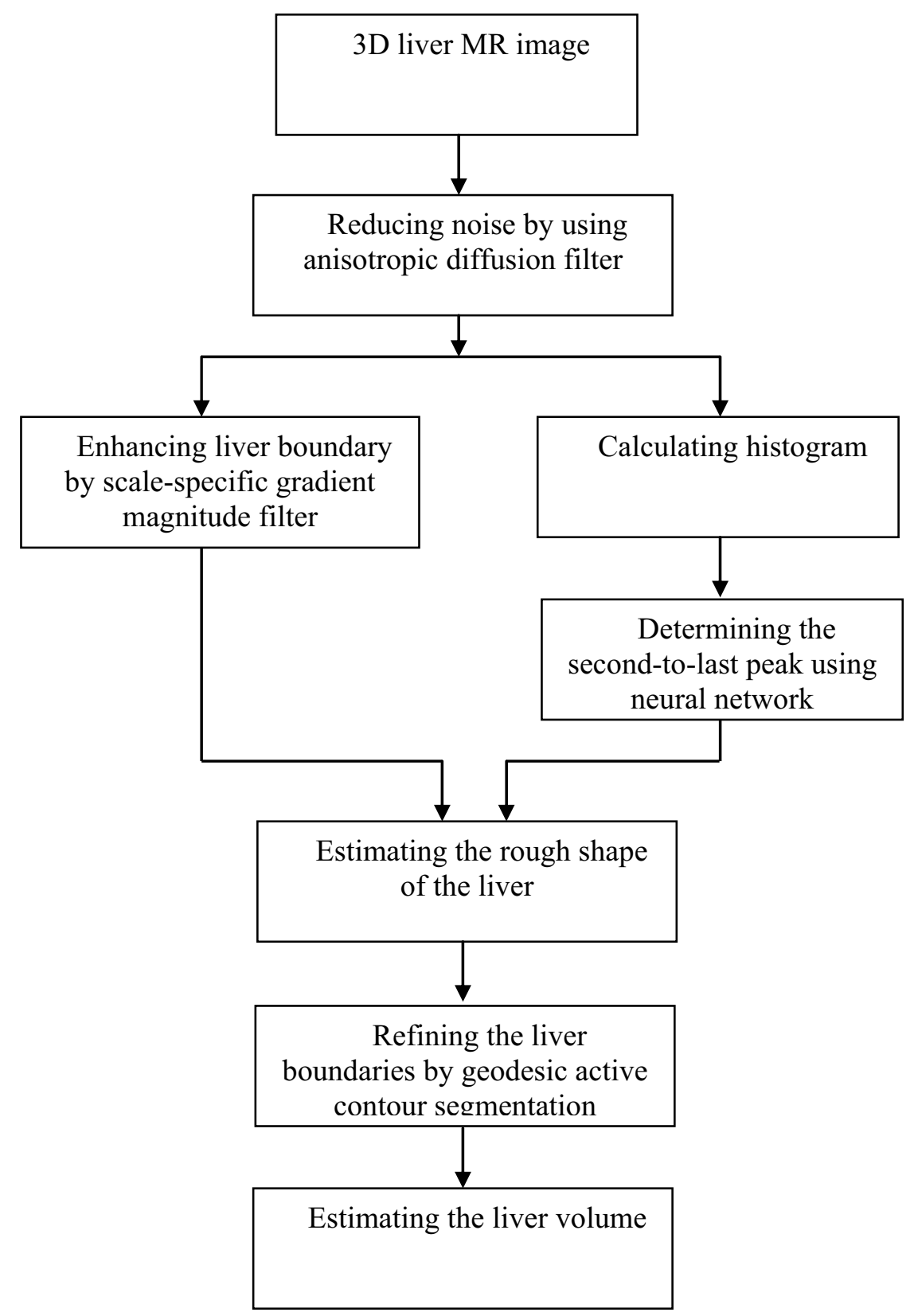

Fig. 1. Overview of the proposed automatic liver volumetry scheme. 
The histogram of the 3D MR image is calculated, and then a generalized regression neural network is applied to smooth the curve, as shown in Figure 2. The peaks of the curve are determined. Let lpk be the intensity value corresponding to the second-to-last peak of histogram; the intensity distribution of the liver is around this value.

The segmentation of the liver from a MR image is accomplished by a two-step process involving rough approximation and precise refinement. First, a thresholding method is employed to create the thresholding image. Two thresholds are calculated by the following:

$$
\begin{aligned}
& \text { lowerThreshold }=l p k-\tau \\
& \text { upperThreshold }=l p k+\zeta,
\end{aligned}
$$

where $\tau$ and $\zeta$ are user-defined parameters (set to 150 in this scheme).

Due to the voxel level similarity, the thresholding image may include other organs from among which the liver has the largest volume. Therefore, in order to separate the liver from any remaining organs, the thresholding image is refined using the magnitude of the image gradient. The value of the refined image at each voxel $\mathbf{p}$ is updated by:

$$
I_{T}(\mathbf{p})=I_{t h}(\mathbf{p}) u\left(I_{M}(\mathbf{p})-\lambda\right)
$$

where $I_{\mathrm{T}}$ is the refined image, $I_{t h}$ is the thresholding image, $\lambda$ is an user-defined parameter (set to 20 in this scheme), and $u$ is the unit step function defined by:

$$
u(x)=\left\{\begin{array}{cc}
1 & \text { if } x \geq 0 \\
0 & \text { otherwise }
\end{array}\right. \text {. }
$$

The opening and connected component operations are applied to the $I_{\mathrm{T}}$ image, and then the largest volume region is filtered out. The closing operation is then applied to this filtered region in order to create the rough shape of the liver. Note that the structuring element used in the opening and closing operations is a ball (with a radius of 1 in this scheme).

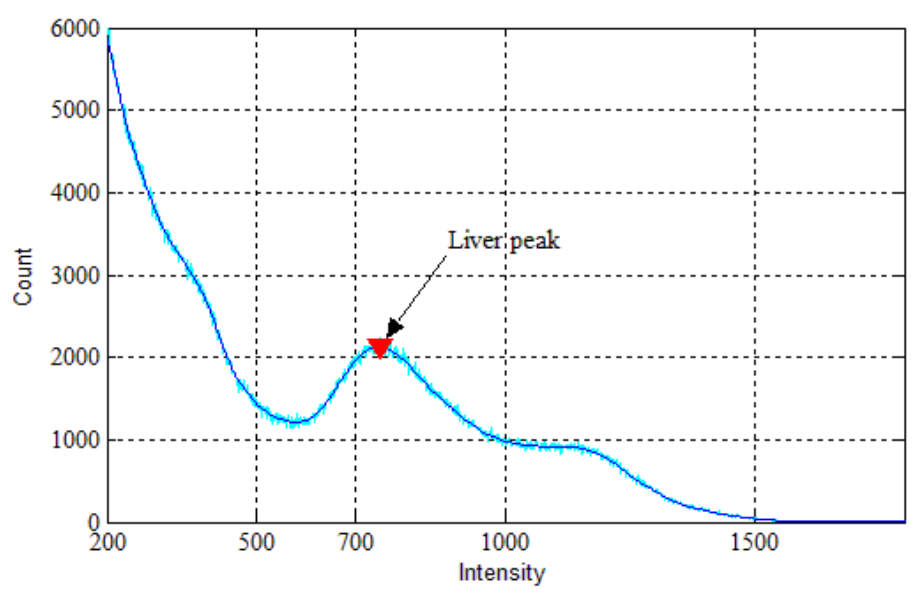

Fig. 2. Histogram of a 3D MR image. 
A geodesic active contour level-set segmentation is applied to refine the initial surface determined by the rough liver shape so as to more precisely determine the liver boundaries. Let $\psi(\mathbf{p}, t)$ be a level set function with the initial surface corresponding to $\psi(\mathbf{p}, t=0)$. The evolution of the level set function is controlled by the partial differential equation [27]:

$$
\frac{d \psi}{d t}=-\alpha A(\mathbf{p}) \cdot \nabla \psi-\beta F(\mathbf{p})|\nabla \psi|+\gamma Z(\mathbf{p}) \kappa|\nabla \psi|,
$$

where $A(\cdot)$ is an advection vector function, $F(\cdot)$ is a propagation (or speed) function, and $Z(\cdot)$ is a spatial modifier function for the mean curvature $\kappa$. The spatial modifier term controls the smoothness of the surface where regions of high curvature are smoothed out. The scalar constants $\alpha, \beta$, and $\gamma$ allow trading off among three terms: advection, propagation, and curvature; in this scheme, $\alpha=1.5, \beta=1.3$, and $\gamma=0.8$. The algorithm requires an initial zero-level set that contains a rough approximation of liver boundaries. In this scheme, the initial zero-level set is obtained from the rough shape of the liver. The approximated surface is propagated with speed and direction (outwards, inwards) that is controlled by the propagation function. This algorithm used the liver-parenchyma-enhanced image obtained from the gradient magnitude image as the propagation (or speed) term for the evolution of the front. With this image, the front moves rather fast in the low-gradient regions and moves very slowly in the highgradient regions. The level set evolution is terminated when either the convergence criterion or the maximum number of iterations is reached.

\section{Experimental results}

\subsection{MRI cases}

The database of this study consisted of 10 cases of 3D MRI scans in the supine position with $1.5 \mathrm{~T}$ MRI scanners (Avanto, Siemens) at the Medic Medical Center, which is one of the largest diagnostic imaging centers in Viet Nam. Post-contrast MR images were obtained by using the T1-weight volumetric interpolated breath-hold examination (VIBE) sequence. A flip angle of 10 degrees was used with $\mathrm{TR}=4.74$ and $\mathrm{TE}=2.38$. The scanning parameters included collimation and reconstruction intervals ranging from 3.5 to $4 \mathrm{~mm}$. Each MRI slice had a matrix size of 230x320 pixels with an inplane pixel size ranging from 1.18 to $1.4 \mathrm{~mm}$.

On each MRI slice that contained the liver, a board-certified abdominal radiologist carefully manually traced the liver contours. The number of slices in each case ranged from 44 to 56 . The liver volume was calculated by multiplying the areas of the manually traced regions in each slice by the reconstruction interval. The total liver volume in each case was determined by the summation of the volumes in all of the slices. The times required to complete the manual contour tracing were recorded.

\subsection{Evaluation criteria}

The liver volumes obtained by the proposed scheme were compared to the "gold-standard" manually determined liver volumes. The true positive $(T P)$, false positive $(F P)$, true negative $(T N)$, and false negative $(F N)$ segmentation were determined for detailed analysis. The segmentation accuracy can be calculated by: 


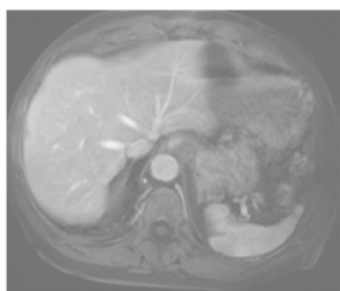

(a)

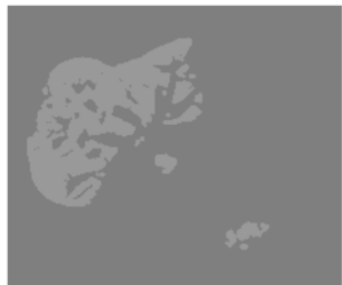

(e)

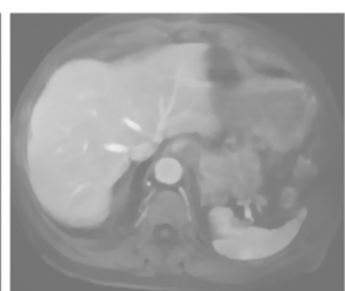

(b)

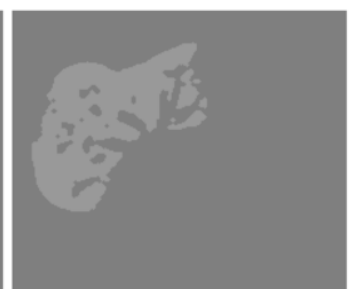

(f)

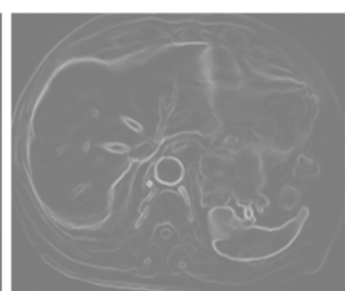

(c)

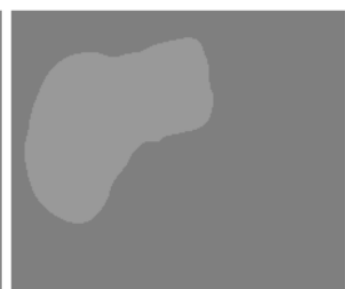

(g)



(d)

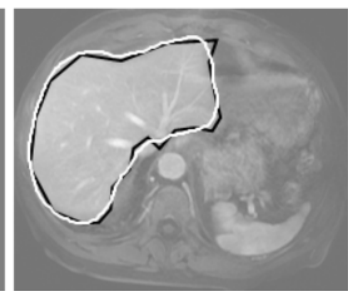

(h)

Fig. 3. 77-year-old man; intermediate results of the proposed scheme for a sample case are shown (slices contain a large region of liver): (a) Original MR image; (b) Anisotropic diffusion noise reduction; (c) Gradient magnitude calculation; (d) Thresholding image; (e) The opening operation using a ball structuring element; (f) The largest volume region extraction (the accuracy and Dice coefficient are $98.2 \%$ and $75.3 \%$, respectively); (g) Geodesic active contour segmentation (the accuracy and Dice coefficient are 99.2\% and 91.6\%, respectively); (h) Comparison between the automatic liver extraction (white contours) and the "gold-standard" manual liver extraction (black contours).

$$
\text { Accuracy }=(|T P|+|T N|) /|I|
$$

where $I$ is the entire image. The Dice measurement representing the fraction of the overlapping volume and the volume of the two segmentation methods is given by:

$$
\text { Dice }=\frac{2|T P|}{2|T P|+|F P|+|F N|},
$$

The percentage volume error $(E)$ for each automated volume $\left(V_{\mathrm{a}}\right)$ and the gold standard manual volume $\left(V_{\mathrm{g}}\right)$ were also defined as:

$$
E=\left|\left(V_{a}-V_{g}\right) / V_{g}\right|
$$

\subsection{Results}

Figure 3 illustrates the intermediate results of the proposed scheme for an example case with slices in which the liver region was large. The noise of the original MR image in Figure 3(a) was reduced by using an anisotropic diffusion filter, as shown in Figure 3(b). A gradient magnitude filter was applied to the reduced-noise image in order to enhance the boundaries and create an edge image, as shown in Figure 3(c). A thresholding method was employed to create the thresholding image, as shown in Figure 3(d). The opening operation using a ball structuring element was applied to the thresholding image (after binarization) to separate the liver from the other organs, as shown in Figure 3(e). The connected component operation was applied and the largest volume region was filtered out, as shown 
in Figure 3(f). The closing operation was applied to generate the rough shape of liver. Finally, the liver was more precisely segmented using the geodesic active contour algorithm, as shown in Figure 3(g). A comparison of the automatic liver segmentation with the "gold-standard" manual liver segmentation is illustrated in Figure 3(h), in which the black contours are from the manually tracing method, and the white contours are from the automatic scheme. The liver volume was computed from the segmented regions.

Figure 4 illustrates intermediate results of the proposed scheme used for another example case with slices in which the liver region was small. The noise of the original image in Figure 4(a) was reduced, and a threshold method was used to create the thresholding image that is shown in Figure 4(b). Figure 4(c) was obtained after applying the geodesic active contour algorithm. A comparison of the automatic liver segmentation with the "gold-standard" manual liver segmentation is illustrated in Figure 4(d), in which the black contours are from the manually tracing method, and the white contours are from the automatic scheme.

Compared to the manually tracing method, the proposed automatic scheme obtained a percentage volume error of $8.7 \%$. The overall mean of the Dice coefficients was calculated at $91.0 \pm 2.8 \%$, and the accuracy of the liver segmentation was $99.0 \pm 0.4 \%$. The average processing time of the proposed liver segmentation scheme was $1.02 \pm 0.8$ min per case on a PC (CPU: Intel, core i7, $2.8 \mathrm{GHz}$ ), whereas that of the manual method was $24.3 \pm 3.7 \mathrm{~min}$ per case. The difference was statistically significant $(\mathrm{p}<$ $0.001)$.

\section{Discussion}

An important factor to consider in hepatectomy is liver volumetry. It is crucial for ensuring the success of a liver transplantation; and for a successful transplant, the recipient must receive a minimum of $40 \%$ of the standard liver mass, whereas the donor must retain $30-40 \%$ of the original volume in order to survive [28]. However, in some complicated cases, such as those involving chronic liver disease, an even greater liver volume is likely required.

To date, researchers have proposed several schemes for CT image-based liver volumetry. However, there remain a limited number of proposed MRI-based liver volumetry schemes. Thus, it is essential to investigate the potential of a computerized volumetry for liver MR images, and it is believed that computerized MR liver volumetry will prove to be highly beneficial in the field.

At present, there are schemes for liver volumetry on MR images based on the fast marching and level set algorithms, but these schemes require initial seed points, and choosing the appropriate seed points remains problematic. Not only is this time consuming, but may also impact the final results.

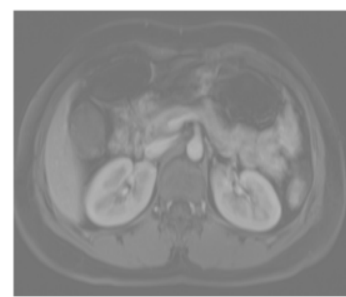

a)

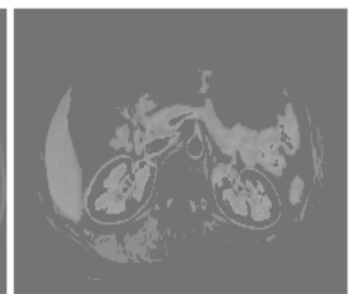

b)

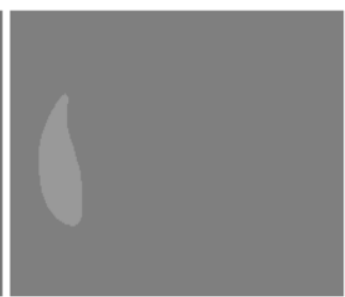

c)

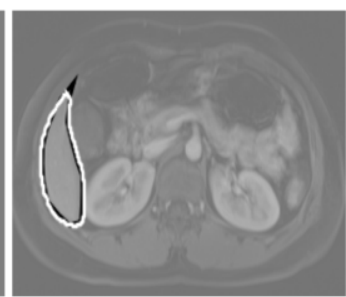

d)

Fig. 4. 37-year-old woman; intermediate results of the proposed scheme for a sample case (slices contain a small region of liver): (a) Original MR image; (b) Thresholding image; (c) Geodesic active contour segmentation; (d) Comparison between the automatic liver extraction (white contours) and the "gold-standard" manual liver extraction (black contours). 
Therefore, it is essential to investigate approaches that reduce user interactions.

The scheme proposed in this study has several parameters. As done in the previous studies, these parameters were determined based on experiments. In this scheme, one case was used to determine the values of these parameters and these values were kept for the remaining cases.

Although the proposed scheme can obtain a high accuracy in comparison to the "gold-standard" manually tracing method, the correlation between the two does not meet the minimal variation obtained in CT volumetry between expert radiologists, which was reported as 0.997 [29]. (Although a study reporting the variation in MR volumetry was not found, it is expected that it would be larger than that in CT). The overall accuracy can be further improved by manually correcting the false negative and false positive segmentations, and this can be accomplished quickly by routine manipulations. The proposed automatic scheme saved a substantial amount of time with a small error rate (an average processing time of $1.02 \mathrm{~min}$ per case with an average percentage volume error of $8.7 \%$ ) compared to the manually tracing method (an average processing time of $24.3 \mathrm{~min}$ per case).

\section{Conclusion}

The increasing use of liver MRI as the single exam for liver resection and transplantation has led to imperative demands for automatic MRI liver volumetry. However, there are currently only a few studies that have explored this challenging task. In the authors' previous study, a scheme was proposed that used the fast marching algorithm and required seed points chosen by users. However, in this study, a fully automatic scheme for liver volumetry in MR images was developed by employing the thresholding methods combined with geodesic active contour segmentation. This approach does not require users to choose seed points. Furthermore, the time required for the proposed volumetry scheme is significantly reduced from 24 minutes per case to just one minute per case. Compared to the "gold-standard" manually tracing method, the proposed scheme can obtain very high accuracy and Dice coefficient.

\section{Acknowledgment}

This research is funded by the Vietnam National Foundation for Science and Technology Development (NAFOSTED) under grant number 102.01-2013.47. The authors are grateful to Phan Thanh Hai, MD, and Nguyen Thanh Dang, MD for preparing images and their clinical advice.

\section{References}

[1] I.R. Kamel, J.B. Kruskal, G. Warmbrand, et al., Accuracy of volumetric measurements after virtual right hepatectomy in potential donors undergoing living adult liver transplantation, American Journal of Roentgenology 176 (2) (2001), 483-487.

[2] R. Emiroglu, M. Coskun, U. Yilmaz, et al., Safety of multidetector computed tomography in calculating liver volume for living-donor liver transplantation, Transplantation Proceedings 38 (10) (2006), 3576-3578.

[3] A.J. Lemke, M.J. Brinkmann, T. Schott, et al., Living donor right liver lobes: preoperative CT volumetric measurement for calculation of intraoperative weight and volume, Radiology 240 (3) (2006), 736-742.

[4] A. Radtke, G.C. Sotiropoulos, S. Nadalin, et al., Preoperative volume prediction in adult living donor liver transplantation: how much can we rely on it? American Journal of Transplantation 7 (3) (2007), 672-679. 
[5] Y. Nakayama, Q. Li, S. Katsuragawa, et al, Automated hepatic volumetry for living related liver transplantation at multisection CT, Radiology 240 (2006), 743-748.

[6] L. Gao, D.G. Heath, B.S. Kuszyk and E.K. Fishman, Automatic liver segmentation technique for three-dimensional visualization of CT data, Radiology 201 (1996), 359-364.

[7] K.T. Bae, M.L. Giger, C.T. Chen, et al., Automatic segmentation of liver structure in CT images, Medical Physics 20 (1993), 71-78.

[8] L. Hermoye, I. Laamari-Azjal, Z. Cao, et al., Liver segmentation in living liver transplant donors: comparison of semiautomatic and manual methods, Radiology 234 (2005), 171-178.

[9] L. Ruskó, G. Bekes, G. Németh and M. Fidrich, Fully automatic liver segmentation for contrast-enhanced CT images, Proceedings of MICCAI Workshop 3-D Segmentation Clinic: A Grand Challenge, 2007, pp. 143-150.

[10] R. Susomboon, D. S. Raicu, and J. Furst, A hybrid approach for liver segmentation. Proceedings of MICCAI Workshop on 3-D Segmentation Clinic: A Grand Challenge, 2007, pp. 151-160.

[11] A. Beck and V. Aurich, HepaTux-a semiautomatic liver segmentation system, Proceedings of MICCAI Workshop on 3D Segmentation Clinic: A Grand Challenge, 2007, pp. 225-234.

[12] D. Kainmüller, T. Lange, and H. Lamecker, Shape constrained automatic segmentation of the liver based on a heuristic intensity model, Proceedings ofMICCAI Workshop 3-D Segmentation Clinic: A Grand Challenge, 2007, pp. 109-116.

[13] T. Heimann, H. P. Meinzer, and I. Wolf, A statistical deformable model for the segmentation of liver CT volumes, Proceedings of MICCAI Workshop 3-D Segmentation in the Clinic: A Grand Challenge, 2007, pp. 161-166.

[14] K. A. Saddi, M. Rousson, C. Chefd'hotel C, and F. Cheriet, Global-to-local shape matching for liver segmentation in CT imaging, Proceedings of MICCAI Workshop 3-D Segmentation Clinic: A Grand Challenge, 2007, pp. 207-214.

[15] D. Seghers, P. Slagmolen, Y. Lambelin, et al., Landmark based liver segmentation using local shape and local intensity models, Proceedings of MICCAI Workshop3-D Segmentation Clinic: A Grand Challenge, 2007, pp. 135-142.

[16] G. Schmidt, M. A. Athelogou, R. Schönmeyer, et al., Cognition network technology for a fully automated 3-D segmentation of liver. Proc. MICCAI Workshop 3-D Segmentation Clinic: A Grand Challenge, 2007, pp. 125-133.

[17] R. Beichel, C. Bauer, A. Bornik, et al., Liver segmentation in CT data: A segmentation refinement approach, Proceedings of MICCAI Workshop 3-D Segmentat. Clinic: A Grand Challenge, 2007, pp. 235-245.

[18] Kenji Suzuki, Ryan Kohlbrenner, Mark L. Epstein, et al., Computer-aided measurement of liver volumes in CT by means of geodesic active contour segmentation coupled with level-set algorithms, Medical Physics 37 (5) (2010), 21592166.

[19] Kenji Suzuki, Mark L. Epstein, Ryan Kohlbrenner, Shailesh Garg, Masatoshi Hori, Aytekin Oto and Richard L. Baron, Quantitative radiology: automated CT liver volumetry compared with interactive volumetry and manual volumetry, Medical Physics and Informatics, AJR 197 (2011), W706-712.

[20] C. Karlo, C.S. Reiner, P. Stolzmann, et al., CT- and MRI-based volumetry of resected liver specimen: comparison to intraoperative volume and weight measurements and calculation of conversion factors, European Journal of Radiology 75 (1) (2010), e107-111.

[21] S.W. Farraher, H. Jara, K.J. Chang, A. Hou and J.A. Soto, Liver and spleen volumetry with quantitative MR imaging and dual-space clustering segmentation, Radiology 237 (2005), 322-328.

[22] L. Rusko and G. Bekes, Liver segmentation for contrast-enhanced MR images using partitioned probabilistic model, International Journal of Computer Assisted Radiology and Surgery 6 (2011), 13-20.

[23] O. Gloger, J. Kuhn, A. Stanski, H. Volzke and R. Puls, A fully automatic three-step liver segmentation method on LDA-based probability maps for multiple contrast MR images, Magnetic Resonance Imaging 28 (2010), 882-897.

[24] Hieu Trung Huynh, Ibrahim Karademir, Aytekin Oto and Kenji Suzuki, Computerized liver volumetry on MRI by using 3D geodesic active contour segmentation, Medical physics and Informatics, American Journal of Roentgenology 202 (2014), 152-159.

[25] R.T. Whitaker and X. Xue, Variable-conductance, level-set curvature for image denoising, Proceedings of International Conference on Image Processing 3 (2001), 142-145.

[26] P. Perona and J. Malik, Scale-space and edge detection using anisotropic diffusion, IEEE Transactions on Pattern Analysis Machine Intelligence 12 (1990), 629-639.

[27] C. Vicen, K. Ron and S. Guillermo, Geodesic active contours, International Journal of Computer Vision 22 (1) (1997), 61-79.

[28] C. M. Lo, S.T. Fan, C.L. Liu, et al., Adult-to-adult living donor liver transplantation using extended right lobe grafts, Annals of Surgery 226 (1997), 261-270.

[29] K. Sandrasegaran, P.W. Kwo, D. DiGirolamo, S.M. Stockberger, Jr., O.W. Cummings and K.K. Kopecky, Measurement of liver volume using spiral CT and the curved line and cubic spline algorithms: reproducibility and interobserver variation, Abdominal Imaging 24 (1999), 61-65. 